\title{
Front-line-Chemotherapie plus Bevacizumab verlängert das progressionsfreie Überleben
}

In einer randomisierten Phase-III-Studie verlängerte die Kombination aus Carboplatin/Paclitaxel plus Bevacizumab mit nachfolgender Bevacizumab-Erhaltungstherapie das progressionsfreie Überleben (PFS) von Patientinnen mit fortgeschrittenem Ovarialkarzinom (Burger RA et al., ASCO 2010: \#LBA1). Mit der zusätzlichen Gabe von Bevacizumab wurde damit erstmals seit Jahren wieder ein Vorteil in der Front-line-Therapie des fortgeschrittenen Ovarialkarzinoms erreicht.

Die GOG-0218-Studie der US-amerikanischen Studiengesellschaft GOG (Gynecologic Oncology Group) ist eine prospektive, doppelblinde, placebokontrollierte Phase-III-Studie mit 1.873 Patientinnen mit fortgeschrittenem, nicht rezidiviertem epithelialem Ovarialkarzinom, primärem Peritonealkarzinom oder primärem Eileiterkarzinom der Stadien III/IV. Die systemisch nicht vorbehandelten Patientinnen wurden postoperativ in 3 Therapiearme randomisiert: Sie erhielten jeweils 6 Zyklen der Standardchemotherapie Carboplatin/Paclitaxel, die in den experimentellen Therapiearmen 2 und $3 \mathrm{ab}$ dem 2. Zyklus in Kombination mit Bevacizumab gegeben wurde. Im Therapiearm 3 erhielten die Patientinnen Bevacizumab zusätzlich für weitere 16 Zyklen als Erhaltungstherapie (Zyklus 7-22). Primärer Endpunkt war das PFS.

Nach einer medianen Nachbeobachtungszeit von 17,4 Monaten (Intention-to-treat-Analyse) zeigte sich beim PFS ein signifikanter Vorteil zugunsten der kombinierten Behandlung aus Chemotherapie/Bevacizumab plus Bevacizumab-Erhaltungstherapie (Arm 3). Im Vergleich zum Kontrollarm ohne Bevacizumab (Arm 1) blieben diese Patientinnen 3,8 Monate länger ohne Progression (median 14,1 Monate vs. 10,3 Monate; Hazard-Ratio [HR] 0,717; $p<0,0001$; Abb.). Der Vorteil beim PFS war unabhängig vom Krankheitsstadium, Allgemeinzustand und Alter der Patientinnen. Im zweiten experimentellen Therapiearm ohne Bevacizumab-Erhaltungstherapie (Arm 2) war der tendenzielle Vorteil beim PFS im Vergleich zum Kontrollarm nicht signifikant (Abb.).

Das Vorliegen einer Progression wurde nach den RECIST-Kriterien und der Bestimmung des Tumormarkers CA-125 im Serum ermittelt. Die CA-125-Bestimmung gilt jedoch nicht als zuverlässiger Surrogatmarker für das Vorliegen einer Progression. In der CA-125-

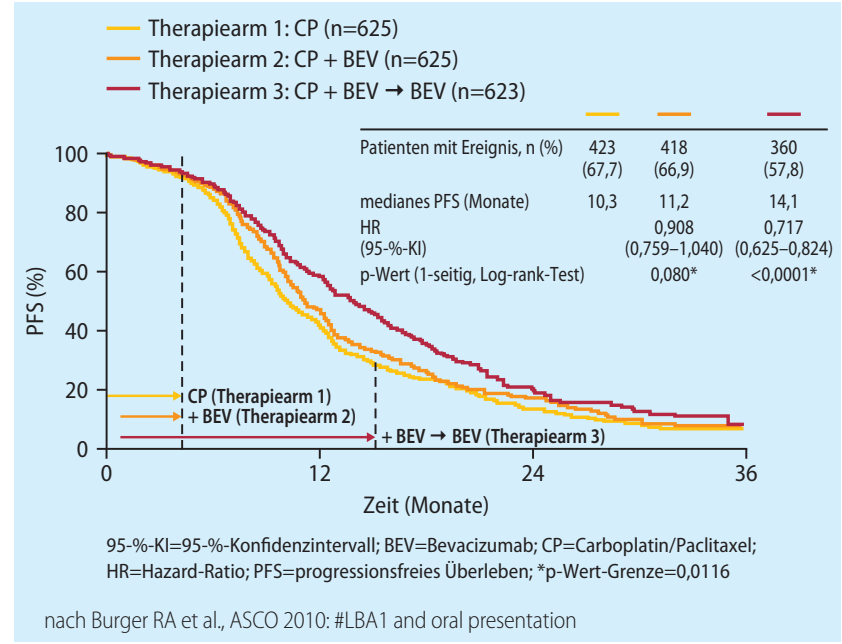

Phase-III-Studie GOG 0218 - progressionsfreies Überleben (PFS)

zensierten Auswertung vergrößerte sich der Vorteil beim PFS zugunsten der kombinierten Behandlung aus Chemotherapie/Bevacizumab plus Bevacizumab-Erhaltungstherapie (Arm 3) verglichen mit dem Kontrollarm auf 6 Monate (median 18 Monate vs. 12 Monate; HR 0,645; p $\leq 0,0001$ ). Aussagekräftige Daten zum Gesamtüberleben liegen noch nicht vor, da zum Auswertungszeitraum 76\% der Patientinnen diesen Endpunkt noch nicht erreicht hatten. Die Hinzunahme von Bevacizumab zur Chemotherapie wurde gut vertragen. Die Inzidenz hämatologischer Nebenwirkungen und gastrointestinaler Ereignisse oder das Blutungsrisiko waren nicht signifikant erhöht. Insgesamt entsprach das Sicherheitsprofil dem der vorangegangenen zulassungsrelevanten Studien mit Bevacizumab. BKP
Axilladissektion bei frühem Mammakarzinom

\section{Kein Vorteil beim Gesamtüberleben}

In der Phase-III-Studie ACOSOG (American College of Surgeons Oncology Group) Z0011 mit 891 Mammakarzinom-Patientinnen (klinisches Stadium T1-2 N0 M0) mit positivem Sentinellymphknoten und günstigem Risikoprofil wurde beim Gesamtüberleben nach median 6,2 Jahren Follow-up kein Unterschied zwischen alleiniger Senti- nellymphonodektomie und zusätzlicher Axilladissektion mit Entfernung von $\geq 10$ Lymphknoten festgestellt (Giuliano AE et al., ASCO 2010: \#CRA506).

Das 5-Jahres-Gesamtüberleben betrug $92,5 \%$ bei alleiniger Sentinellymphonodektomie und $91,8 \%$ bei zusätzlicher Axilla- dissektion $(p=0,25)$. Auch das krankheitsfreie Überleben und die lokoregionäre Kontrollrate waren nicht signifikant unterschiedlich. Alle Patientinnen waren brusterhaltend operiert worden und hatten eine Bestrahlung der Mamma erhalten. Eine adjuvante systemische Therapie war nach Ermessen des behandelnden Arztes erfolgt. Die Ergebnisse stützen laut Giuliano nicht die routinemäßige Axilladissektion bei MammakarzinomPatientinnen mit Mikro- und Makrometastasen im Sentinellymphknoten. $A M$ 